\title{
Araucaria Project: Pulsating stars in binary systems and as distance indicators
}

\author{
Bogumił Pilecki ${ }^{1, \star}$, Wolfgang Gieren ${ }^{2,3, \star \star}$, Grzegorz Pietrzyński ${ }^{1}$, and Radosław Smolec ${ }^{1}$ \\ ${ }^{1}$ Copernicus Astronomical Centre, Polish Academy of Sciences, Bartycka 18, 00-716 Warsaw, Poland \\ ${ }^{2}$ Universidad de Concepción, Departamento de Astronomía, Casilla 160-C, Concepción, Chile \\ ${ }^{3}$ Millennium Institute of Astrophysics, Santiago, Chile
}

\begin{abstract}
Pulsating stars, like Cepheids or RR Lyrae stars, are ones of the most important distance indicators. They are also key objects for testing the predictions of stellar evolution and stellar pulsation theory. In the Araucaria Project we have studied these objects since 2002, measuring distances to the galaxies in the Local Group and beyond.

In 2010 we have for the first time confirmed spectroscopically the existence of a classical Cepheid in an eclipsing binary system. This has opened an opportunity to study in great details and with high accuracy (better than $1 \%$ ) the physical parameters of these very important objects. First dynamical mass determination $\left(M_{c e p}=4.16 \pm 0.03 M_{\odot}\right)$ let us solve the long-standing mass discrepancy problem. Since then we have measured masses for 6 classical Cepheids in binary systems and determined projection factors for three of them. One of the analyzed systems was confirmed to consist of two first-overtone Cepheids.

Type II Cepheids are recently becoming more important as distance indicators and astrophysics laboratory, although our knowledge of these stars is quite limited. Their evolutionary status is also not well understood and observational constraints are needed to confirm the current theories. We are presenting here our first results of the spectroscopic analysis of 4 of these systems. The masses of type II Cepheids seem consistent with the expected $0.5-0.6 M_{\odot}$. We also present first results of the fully modeled pulsator originally classified as peculiar W Vir star. The mass of this star is $1.51 \pm 0.09 M_{\odot}$ and the p-factor $1.3 \pm 0.03$. It was eventually found not to belong to any typical Cepheid group.
\end{abstract}

\section{Introduction}

The Araucaria Project, started in 2000, is a collaboration between astronomers from institutions in Chile, the United States and Europe. Its principal aim is to provide an improved calibration of the local extragalactic distance scale. In the process of setting up the extragalactic distance scale, the greatest difficulty leading to the currently largest contribution on the systematic uncertainty of the Hubble constant lies in the determination of accurate absolute distances to nearby galaxies.

The principal reason for this persisting difficulty is in the ill-known dependencies of stellar standard candles, used to measure the distances of nearby galaxies, on the environmental properties of their host galaxies (metallicity, age of the stellar populations).

\footnotetext{
${ }^{\star}$ pilecki@camk.edu.pl

${ }^{\star}$ wgieren@astro-udec.cl
} 
Table 1. Distance measurements utilizing P-L relation for classical Cepheids.

\begin{tabular}{lll}
\hline Galaxy & Distance modulus & Reference \\
\hline M33 & $24.62 \pm 0.07$ & 2013, ApJ, 773, 69 \\
NGC 7793 & $27.68 \pm 0.05$ (stat) \pm 0.08 (sys) & 2010, AJ, 140, 1475 \\
NGC 247 & $27.644 \pm 0.036$ & 2009, ApJ, 700, 1141 \\
NGC 247 & $27.80 \pm 0.09$ (stat) \pm 0.09 (sys) & 2008, AJ, 136, 1770 \\
WLM & $24.924 \pm 0.042$ (stat) & 2008, ApJ, 683, 611 \\
NGC 55 & $26.434 \pm 0.037$ (stat) & 2008, ApJ, 672, 266 \\
WLM & $25.144 \pm 0.03$ (stat) \pm 0.07 (sys) & 2007, AJ, 134, 594 \\
NGC 55 & $26.40 \pm 0.05$ (stat) \pm 0.09 (sys) & 2006, AJ, 132, 2556 \\
NGC 3109 & $25.571 \pm 0.024$ & 2006, ApJ, 648, 375 \\
NGC 3109 & $25.54 \pm 0.03$ & 2006, ApJ, 648, 366 \\
NGC 6822 & $23.312 \pm 0.021$ (stat) & 2006, ApJ, 647, 1056 \\
IC1613 & $24.291 \pm 0.035($ stat) & 2006, ApJ, 642, 216 \\
NGC 300 & $26.37 \pm 0.05$ (stat) \pm 0.03 (sys) & 2005, ApJ, 628, 695 \\
LMC & $18.56 \pm 0.04$ & 2005, ApJ, 627, 224 \\
NGC 6822 & $23.34 \pm 0.04$ (stat) \pm 0.05 (sys) & 2004, AJ, 128, 2815 \\
NGC 300 & $26.43 \pm 0.04$ (stat) \pm 0.05 (sys) & 2004, AJ, 128, 1167 \\
\hline
\end{tabular}

The Araucaria Project is an effort to remedy this situation for several of the most important stellar candles, including Cepheid variables, RR Lyrae stars, red clump giants, and blue supergiants which all have the potential to provide accurate distance determinations to nearby galaxies once their environmental dependencies are well calibrated.

\section{Pulsating stars as distance indicators}

Here we present a short summary of the distances to other galaxies measured by the Araucaria Project using the pulsating stars as standard candles. Such measurements are subject to many systematic errors with the reddening being one of the most significant sources thereof. The differences in distance determination may be as high as 30\%, as in the case of M33 ([1]). One of the cures for this situation is using a distance measurement method that is as reddening independent as possible. In Araucaria Project we perform deep infrared observations of the galaxies and do a multi-wavelength (expressed as total to selective extinction $R$ ) distance moduli fit like the one presented in Figure 3 of [1]. Extrapolating the fit to the value of $R=0$ we obtain the true distance modulus, and the slope of the fit gives us the average total extinction. Distance moduli determined in this way are also quite independent of the applied reddening law.

In Table 1 we present a list of 16 distances to 10 galaxies obtained using the period-luminosity (P-L) relation for classical Cepheids. In total more than 600 Cepheids were observed in optical and more than 300 in infrared bands. In Table 2 we present a similar list with the distances obtained using the P-L relations for type II Cepheids and RR Lyrae stars. As one can see, classical Cepheids are the most important and most widely used standard candle, as being very luminous they can be seen out to large distances. Nevertheless, lower-mass and older type II Cepheids and RR Lyrae stars offer not only a possibility to study population effects when more than one distance indicator can be observed, but sometimes may be the only way to measure the distance to galaxies where classical Cepheids are not present. 
Table 2. Distance measurements utilizing P-L relations for type II Cepheids and RR Lyraes.

\begin{tabular}{|c|c|c|}
\hline Galaxy & Distance modulus & Reference \\
\hline \multicolumn{3}{|c|}{ Type II Cepheids: } \\
\hline SMC & $18.85 \pm 0.07$ (stat) \pm 0.07 (sys) & 2010, AcA, 60, 233 \\
\hline \multicolumn{3}{|c|}{ RR Lyraes: } \\
\hline Carina & $20.12 \pm 0.02$ (stat) \pm 0.11 (sys) & 2015, AJ, 150, 3 \\
\hline SMC & $18.97 \pm 0.03($ stat $) \pm 0.12($ sys $)$ & 2009, AJ, 138, 1661 \\
\hline LMC & $18.58 \pm 0.03$ (stat) \pm 0.11 (sys) & $2008, \mathrm{AJ}, 136,272-279$ \\
\hline SDG & $19.67 \pm 0.02$ (stat) \pm 0.12 (sys) & 2008, AJ, 135, 1993-1997 \\
\hline
\end{tabular}

\section{Pulsating stars in eclipsing binary systems}

The results presented above are very important, but such studies utilize the pulsating stars, not telling us anything about the physics behind. To get to know all the parameters of the stars and to study the astrophysics of Cepheids and other pulsators it is very important to analyze this type of stars which are members of eclipsing binary systems. If the system is not eclipsing it can be studied with joint spectroscopic and interferometric observations, but in this case the system has to be located very close to us and have reasonably short orbital period. Up to now no system could be precisely analyzed in this way.

On the other hand, more than ten eclipsing systems with Cepheids were detected so far, and we have analyzed several of them already. The summary of our studies of these systems is presented below.

\subsection{Classical Cepheids}

Classical Cepheids are radially pulsating giants and supergiants of Population I. Thanks to their period-luminosity relation they are important distance indicators in the local Universe. They form a fundamental part of the cosmic distance ladder, connecting our Milky Way galaxy to galaxies in the Local Group and beyond. They are also key objects for testing the predictions of stellar evolution and stellar pulsation theory. Until recently we did not know their physical properties, however, and there are still many challenges to be faced.

Although Cepheids in binary systems were known to exist, for many years it was hard to find one that would be a member of an eclipsing binary. The breakthrough came with the microlensing surveys like OGLE ([2]) and MACHO ([3]). More than ten candidates in the Small (SMC) and Large (LMC) Magellanic Clouds were proposed after the analysis of their light curves; spectroscopic confirmation was however needed to remove all the doubts. We have then selected the most promising objects and observed them spectroscopically.

OGLE-LMC-CEP-0227 was the first to be confirmed ([4]). It was later reanalyzed by [5] using a more advanced method and more data. Five other Cepheids were confirmed and their parameters measured until now: OGLE-LMC-CEP-1812 ([6]), OGLE-LMC-CEP-1718 A and B ([7]), OGLELMC-CEP-2532 ([8]) and OGLE-LMC562.05-9009 ([9]).

For detailed description of the method and the analysis steps we refer the reader to [5] and the papers on other Cepheids that follow. Here we will present just a short summary of the subject.

Radial velocities (RV) were measured using the Broadening Function method implemented in the RaveSpan code $([5,10])$. Orbital solutions were then obtained using the same program and sometimes also with the Wilson-Devinney code ([11]) as well. In RaveSpan the pulsations are included and for the optional analysis with the Wilson-Devinney code they were first subtracted. As a result we 


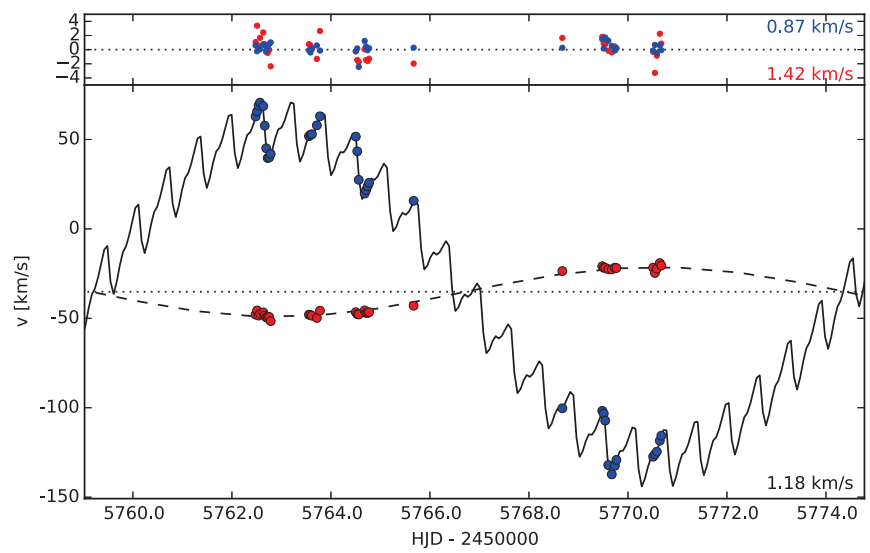

Figure 1. Example radial velocity curve including pulsations and the model obtained with the RaveSpan code.

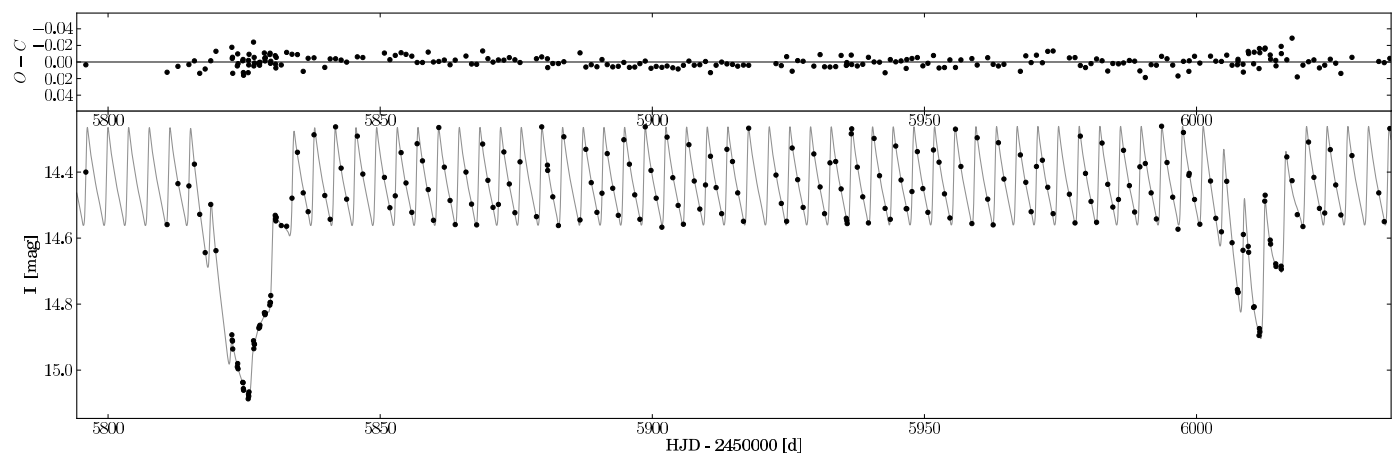

Figure 2. Light curve model of OGLE-LMC-CEP-0227. At the left can be seen the deeper eclipse when the Cepheid is covered by its companion's disk and the amplitude decreases.

have the masses $(M)$ and the system size (scaled by the inclination), the orbital parameters and the pulsational RV curve. The latter is used to measure the radius change after scaling it with the p-factor. Sample radial velocity curve (with the pulsations included) obtained with the RaveSpan is presented in Figure 1.

The photometric data were analyzed using a pulsation-enabled eclipsing modeling tool based on the well-tested JKTEBOP code ([12]) modified to allow the inclusion of pulsation variability. We generate a two-dimensional light curve that consist of purely eclipsing light curves for different pulsating phases. Then a one-dimensional light curve is generated (interpolated from the grid) using a combination of pulsational and orbital phases calculated using a Cepheid and system ephemerides. A model for OGLE-LMC-CEP0227 and its I-band light curve are shown in Figure 2.

From the photometric solution we have the period, the time of the primary minimum, the inclination $(i)$, the fractional radii, the eccentricity, the argument of periastron, the surface brightness ratios, the third light (if present), and the p-factor $(p)$ value. Limb darkening parameters corresponding to 
Table 3. Masses and radii of Cepheids

\begin{tabular}{cccccl}
\hline ID & Type & Period [d] & Mass $\left[M_{\odot}\right]$ & Radius $\left[R_{\odot}\right]$ & Comment \\
\hline LMC-CEP-227 & FU & 3.797086 & $4.16(3)$ & $34.9(2)$ & p-factor \\
LMC562.05-9009 & FU & 2.987846 & $3.71(3)$ & $28.6(2)$ & p-factor \\
LMC-CEP-2532 & 1O & 2.035349 & $3.90(10)$ & $28.9(14)$ & single eclipse \\
LMC-CEP-1718B & 1O & 2.480917 & $4.15(5)$ & $30.0(13)$ & single eclipse \\
LMC-CEP-1718A & 1O & 1.963663 & $4.18(5)$ & $25.3(11)$ & single eclipse \\
LMC-CEP-1812 & FU & 1.312903 & $3.74(6)$ & $17.4(9)$ & prel. p-factor \\
\hline
\end{tabular}
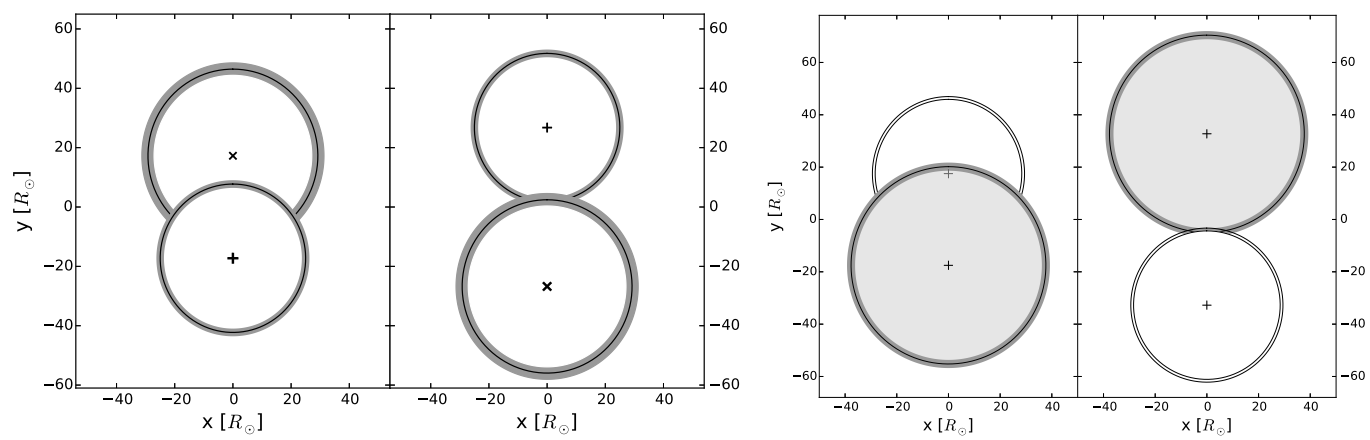

Figure 3. Orbital configuration at the phases of primary and supposed secondary eclipse for OGLE LMC-CEP1718 and LMC-CEP-2532.

different temperatures are also tested. To obtain an optimal solution and error estimation standard Monte Carlo and Markov Chain Monte Carlo (MCMC) sampling is used.

As for now five systems were analyzed yielding physical parameters for three fundamental (FU) and three first-overtone (1O) Cepheids (see the list in Table 3). Using the obtained mass of the OGLELMC-CEP0227 Cepheid $\left(M_{c e p}=4.16 \pm 0.03 M_{\odot}\right)$ we showed that the pulsation theory predicts it correctly and that the theory of evolution has to be adjusted. Expected pulsation mass was $3.98 \pm$ $0.29 M_{\odot}$ and the evolutionary mass suggested masses between 4.5 and $5 M_{\odot}$. The parameters of all analyzed Cepheids can be found in the tables in their corresponding papers (see references above).

First direct, distance-independent p-factor measurements were obtained for two of the stars. For OGLE-LMC-CEP0227 we measured $p=1.21 \pm 0.05$ and for OGLE-LMC562.05-9009 $-p=1.37 \pm$ 0.09. A preliminary p-factor was also obtained for OGLE-LMC-CEP-1812 (paper in prep.), yielding $p=1.27 \pm 0.09$. As the covered period range is short we cannot exclude the period dependence, but the high scatter of our measurements suggest that other factors may have a bigger impact. For the moment anti-correlation with the mass is the clearest one with the higher mass stars having lower p-factors. For the first two stars mentioned the limb darkening was also estimated and the values resulted to be much lower and the phase dependence significantly different than expected.

The OGLE-LMC-CEP-1718 is especially interesting as both stars are pulsating. This allows us not only to constrain the models with the obtained parameters, but also with the difference or ratio of the parameters (their periods for example). What is also interesting is that the masses of the stars are practically equal.

The first-overtone Cepheids are however particularly unlucky as in both cases only one eclipse is present and it is much harder to precisely determine the parameters. In case of OGLE-LMC-CEP- 


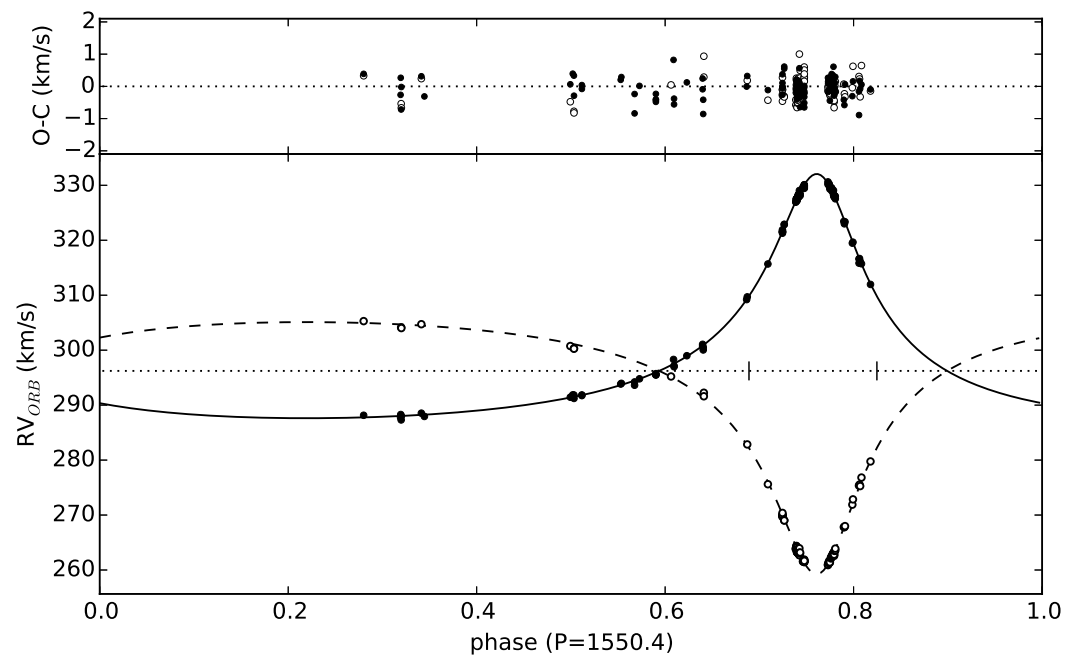

Figure 4. Orbital radial velocity solution for OGLE-LMC562.05-9009. Note the very long period and high eccentricity. The position of eclipses are marked with short vertical lines.

1718 a period-radius relation was used to determine the radii ratio of the stars and only the radii sum was fitted. In case of OGLE-LMC-CEP-2532 we could use the additional information carried by the pulsations, which are directly fitted in our method. The primary eclipse is also much deeper that in case of the former system. The configurations at the times when eclipses should occur for OGLE-LMC-CEP-1718 and OGLE-LMC-CEP-2532 are shown in Figure 3.

The latest analyzed system OGLE-LMC562.05-9009 is very promising, but its long orbital period (1550 days) makes the observational part very challenging. Very high eccentricity $(\sim 0.61)$ makes this task even harder. Nevertheless we could obtain a very precise mass determination $\left(M_{c e p}=3.71 \pm\right.$ $\left.0.03 M_{\odot}\right)$ and another direct measurement of the p-factor value $(1.37 \pm 0.09)$. We could also estimate the age of the Cepheid to be about 205 Myr. It is the lowest mass classical Cepheid found so far, and has the highest p-factor among the three stars for which we could measure it. The orbital solution of OGLE-LMC562.05-9009 is presented in Figure 4. We plan to keep observing this object and reanalyze it once enough new data to significantly improve the model is available.

It is interesting to note that with only one exception all analyzed systems with classical Cepheids have mass ratios in the range of 0.97 to 1.0. Only for OGLE-LMC-CEP-1812, the mass ratio is 0.7, making this system a very interesting object to study. A merger scenario was suggested for this system to explain its current properties ([13]).

\section{Type II Cepheids}

Type II Cepheids are low-mass $\left(\sim 0.5 M_{\odot}\right)$ pulsating stars that belong to the disc and halo populations ([14]). They are a much older counterpart of more massive classical Cepheids - they have periods and amplitudes in a similar range, but are about 1.5-2 mag fainter. Similarly they exhibit a tight and well-defined P-L relation and may serve as a good distance indicators, allowing to measure distances both inside and outside our Galaxy ([15-17]). 


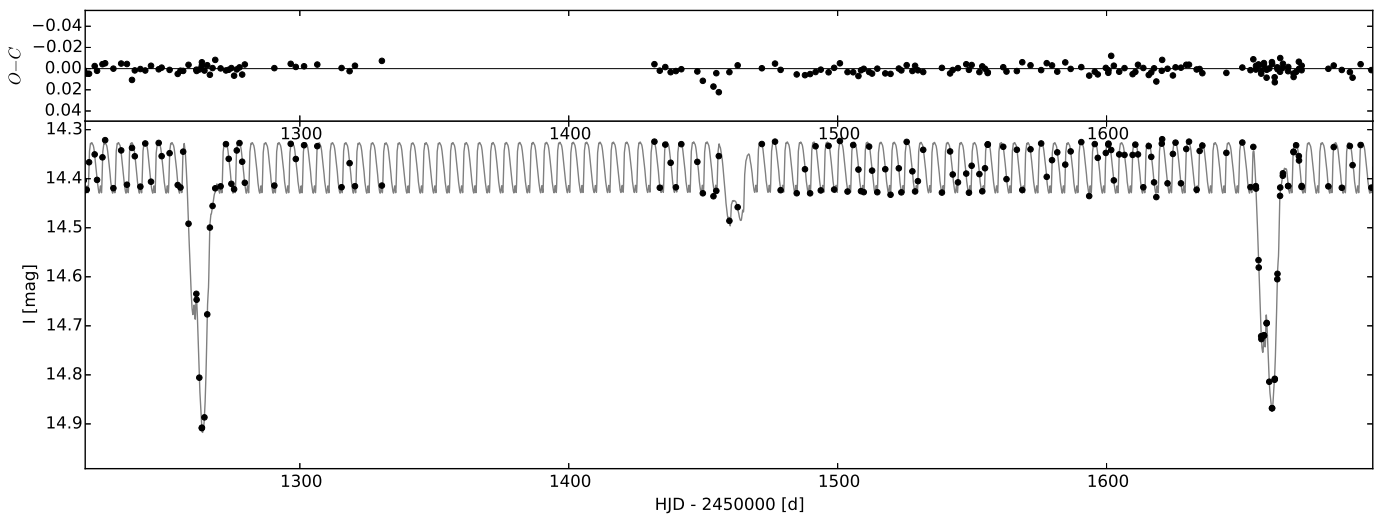

Figure 5. I-band model and OGLE data for OGLE-LMC-T2CEP-098. Note the high difference in the minima depths. The pulsating component is eclipsed at the shallower eclipse.

As compared to classical Cepheids, our knowledge of type II Cepheids is very poor, being more qualitative than quantitative. After the work of Gingold $([18,19])$ they are usually divided into three subgroups (BL Herculis, W Virginis and RV Tauri) depending on the pulsation period, observational properties and their evolutionary status, that nevertheless obey a similar period-luminosity relation. There is however a lack of observational measurements for type II Cepheids that could support the scenario presented by Gingold.

It is now of the highest importance to obtain direct mass measurements for a sample of type II Cepheids to pinpoint their evolutionary status, which is still poorly understood. To measure their masses we need spectroscopic observations of type II Cepheids in eclipsing binary systems. A list of candidates for such systems was given by [16]. We are now collecting observations for 5 systems in LMC and a few more in SMC. Unfortunately only 3 of these systems are clear double-lined spectroscopic binaries.

These systems appear to be very different from the ones that contain classical Cepheids, and much harder to analyze. They have highly unequal minima depths (see Fig. 5) and disk presence is a very common feature. For some of the Cepheids rapid period change was also detected.

We started the analysis with the brightest system that had the best quality light curve, although unfortunately it was of SB1 type. We have obtained the photometric (see Fig. 5) solution and partial orbital solution. The Cepheid companion could not be identified in the spectra, but on the other hand appeared to be many times brighter than the pulsating star. Our method gave us also a tight relation between the p-factor and the mass, i.e. assuming the p-factor we could estimate the mass. This relation limited the mass to the range $0.7-2.4 M_{\odot}$, with the most probable value close to $1.5 M_{\odot}$. Then we have used three mostly independent methods to constrain further the parameters. All of them pointed to a Cepheid mass of about $1.5 M_{\odot}$, with the pulsation theory giving the smallest uncertainty $\left(M_{\text {cep }}=1.51 \pm 0.09 M_{\odot}\right)$. To obtain this result we have used the mass-radius relation obtained from the system modeling. Observable properties of this pulsator makes it an outlier, that does not fit into any known variable type. The most probable explanation is that the star has passed through the mass transfer phase during its evolution. More details on the analysis and the derivation of physical parameters for this system can be found in [20].

The light curves of other systems with type II Cepheids are not easy to model, but have the advantage of being of SB2 type. For them we could obtain a preliminary spectroscopic solution. 
All three measured $M \sin ^{3}(i)$ are located in the range $0.3-0.75 M_{\odot}$. As the systems are detached, we do not expect very low inclinations, thus the masses of these Cepheids will not be much higher than the quoted numbers. As the theory expects masses of about $0.5-0.6 M_{\odot}$, for these cases we see a much better agreement with observations than in case of OGLE-LMC-T2CEP-098. However more detailed studies are necessary to classify correctly these pulsators, as their light curves exhibit features that are not typical for W Virgnis nor BL Herculis stars. In the catalog of the OGLE project ([16]) some of them were classified as peculiar W Virginis, but even among them there are differences.

Contrary to what we found for the systems with classical Cepheids, the measured mass ratios for those with type II Cepheis are very high, being in the range of $M_{2} / M_{c e p} \sim 3-10$.

Acknowledgments: We gratefully acknowledge financial support for this work from the Polish National Science Center grant SONATA 2014/15/D/ST9/02248.

\section{References}

[1] W. Gieren, M. Górski, G. Pietrzyński, P. Konorski, K. Suchomska, D. Graczyk, B. Pilecki, F. Bresolin, R.P. Kudritzki, J. Storm et al., ApJ, 773, 69 (2013), 1305 . 4258

[2] A. Udalski, M.K. Szymański, G. Szymański, AcA, 65, 1 (2015), 1504.05966

[3] C. Alcock, R.A. Allsman, D.R. Alves, A.C. Becker, D.P. Bennett, K.H. Cook, A.J. Drake, K.C. Freeman, K. Griest, S.L. Hawley et al., ApJ, 573, 338 (2002), astro-ph/0201481

[4] G. Pietrzyński, I.B. Thompson, W. Gieren, D. Graczyk, G. Bono, A. Udalski, I. Soszyński, D. Minniti, B. Pilecki, Nature, 468, 542 (2010)

[5] B. Pilecki, D. Graczyk, G. Pietrzyński, W. Gieren, I.B. Thompson, W.L. Freedman, V. Scowcroft, B.F. Madore, MNRAS, 436, 953 (2013), 1308.5023

[6] G. Pietrzyński, I.B. Thompson, D. Graczyk, W. Gieren, B. Pilecki, A. Udalski, I. Soszynski, G. Bono, P. Konorski, N. Nardetto et al., ApJL, 742, L20 (2011), 1109. 5414

[7] W. Gieren, B. Pilecki, G. Pietrzyński, D. Graczyk, I.B. Thompson, I. Soszyński, P. Konorski, R. Smolec, A. Udalski, N. Nardetto et al., ApJ, 786, 80 (2014), 1403. 3617

[8] B. Pilecki, D. Graczyk, W. Gieren, G. Pietrzyński, I.B. Thompson, R. Smolec, A. Udalski, I. Soszyński, P. Konorski, M. Taormina et al., ApJ, 806, 29 (2015), 1504 . 04611

[9] W. Gieren, B. Pilecki, G. Pietrzyński, D. Graczyk, A. Udalski, I. Soszyński, I.B. Thompson, P.G. Prada Moroni, R. Smolec, P. Konorski et al., ApJ, 815, 28 (2015), 1511.02826

[10] B. Pilecki, P. Konorski, M. Gorski, RaveSpan - Radial Velocity and Spectrum Analyzer, in IAU Symposium, edited by M.T. Richards, I. Hubeny (2012), Vol. 282, pp. 301-302

[11] R.E. Wilson, E.J. Devinney, ApJ, 166, 605 (1971)

[12] J. Southworth, P.F.L. Maxted, B. Smalley, MNRAS, 351, 1277 (2004), astro-ph/0403572

[13] H.R. Neilson, R.G. Izzard, N. Langer, R. Ignace, A\&A, 581, L1 (2015), 1508.02725

[14] G. Wallerstein, PASP, 114, 689 (2002)

[15] N. Matsunaga, M.W. Feast, I. Soszyński, MNRAS, 413, 223 (2011), 1012.0098

[16] I. Soszyński, A. Udalski, M.K. Szymański, M. Kubiak, G. Pietrzyński, Ł. Wyrzykowski, O. Szewczyk, K. Ulaczyk, R. Poleski, AcA, 58, 293 (2008), 0811. 3636

[17] D. Majaess, D. Turner, D. Lane, AcA, 59, 403 (2009), 0909. 0181

[18] R.A. Gingold, ApJ, 204, 116 (1976)

[19] R.A. Gingold, Mem. Soc. Astron. Italiana, 56, 169 (1985)

[20] B. Pilecki, W. Gieren, R. Smolec, G. Pietrzyński, I.B. Thompson, R.I. Anderson, G. Bono, I. Soszyński, P. Kervella, N. Nardetto et al., ApJ 842, 110 (2017) 